\title{
Topology Optimization of 3-DOF Peristaltic Structure Robot Based on Vector Continuous Mapping Matrix
}

\author{
Gao Wang, ${ }^{1}$ Dachang Zhu, ${ }^{2}$ and Ning Liu' \\ ${ }^{1}$ School of Information Science and Technology, Jinan University, Guangzhou, Guangdong 510632, China \\ ${ }^{2}$ School of Mechanical and Electrical Engineering, Guangzhou University, Guangzhou, Guangdong 510006, China \\ Correspondence should be addressed to Dachang Zhu; zdc98998@163.com
}

Received 24 February 2016; Accepted 17 August 2016

Academic Editor: Salvatore Strano

Copyright (c) 2016 Gao Wang et al. This is an open access article distributed under the Creative Commons Attribution License, which permits unrestricted use, distribution, and reproduction in any medium, provided the original work is properly cited.

\begin{abstract}
A mechanism for topology optimization of 3-DOF parallel peristaltic structure robot with vector continuous mapping matrix using Solid Isotropic Material with Penalization (SIMP) method is presented in this paper. We focus on how to prevent the differential motion consistency between parallel prototype mechanisms with peristaltic structure. As the conventional parallel robot joints/hinges are no longer needed after topology optimization, therefore, we renamed this kind of 3-DOF robot structures as peristaltic structure. In the proposed method, the vector continuous mapping matrix is built as stress/strain transfer direction conditions for topology optimization of peristaltic structure, and SIMP method is used for multi-inputs and multioutputs decided by parallel prototype mechanisms. Some numerical examples are presented to illustrate the validity of the proposed method.
\end{abstract}

\section{Introduction}

Substantial researchers have been spent on the design of micro-/nanoequipment in recent years. Flexure joints are typically manufactured monolithically, therefore avoiding assembly errors. In terms of operation, flexure joints have little friction loss and do not require lubrication. They generate smooth and continuous displacement without backlash. So flexure joints are usually used to compose the structure of micro-/nanoequipment named as compliant mechanisms $[1,2]$. However, compliant mechanisms cannot finish spatial multidimensional motion characteristics. To overcome the shortcoming, the structure of parallel mechanisms with flexure joints is investigated called compliant parallel mechanism. Yun and Li [3] presented the design and modeling of a new 6-DOF 8-PSS/SPS compliant dual redundant parallel robot with wide-range flexure joints, and the kinematics model of the macro parallel mechanism system via the stiffness model and Newton-Raphson method are adapted to build the dynamic's model for the micromotion system. Dong et al. [4] proposed a compliant ultraprecision parallel positioner based on the coarse/fine dual architecture, and flexure hinges are adopted as compliant passive joints. Choi et al. [5] proposed a compliant parallel mechanism for two translations and applied it to $X Y$ fine motion stage driven by piezo actuators, and four flexure-based prismatic joint chains are arranged in four sides of a target platform to implement the compliant parallel mechanism. Gao and Zhang [6] designed a novel three-DOF compliant parallel mechanism and its performance characterization is analyzed which affects the application potential. Treatments of the characterization and design of compliant parallel mechanisms may also be found in literatures [7-9]. By using the flexure hinges instead of the conventional rigid joints, although this kind of structure composed method has spatial multidimensional motion characteristics, the whole stiffness is decent obviously. It is important to design a perfect structure satisfying the two subjects: high stiffness and multidimensional operation ability. In terms of high stiffness, topology optimization method is proposed for compliant mechanism. The advantages of topology optimization approach are that it does not require a rigid-link mechanism configuration as a starting point and it can be used to design singlepiece fully compliant mechanisms. Ever since Bends $\Phi_{e}$ introduced the homogenization method [10], many topology optimization methods have been developed such as the solid 


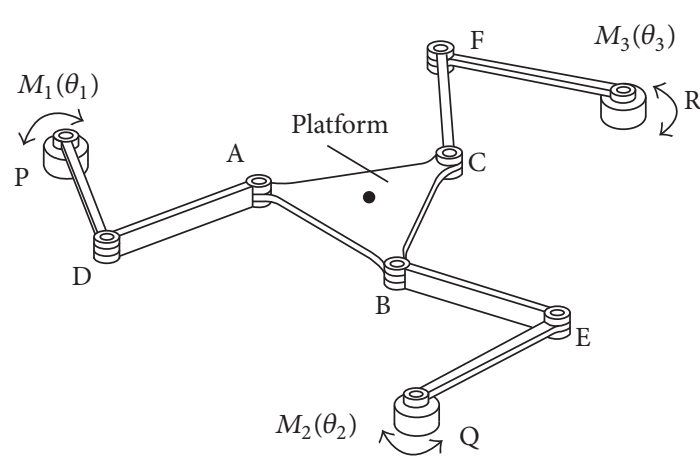

(a)

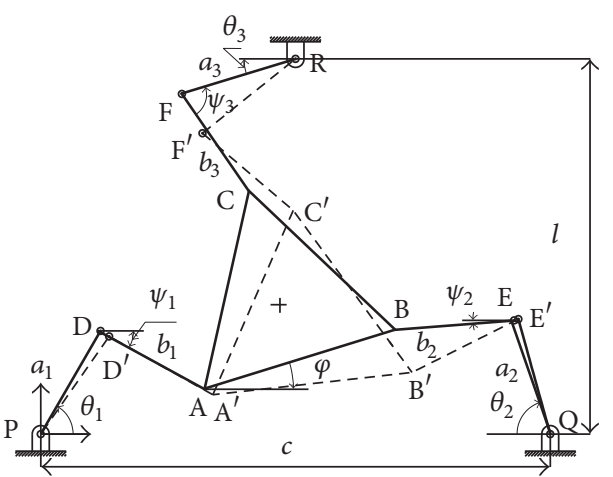

(b)

FIGURE 1: 3-RRR planar parallel prototype mechanism: (a) 3D model of 3-RRR; (b) description of 3-RRR kinematics.

isotropic material with the penalization (SIMP) method [11], the ground structure method [12], and level set based method [13]. Although topology optimization has been a matured tool for optimization design problem, most of topology optimization methods are focusing on the single input-output condition. In reality, parallel compliant mechanisms with multiple inputs and multiple outputs are widely used in the fields of micropositioning and micromanipulation. The relationship between multiple inputs and multiple outputs is vector mapping matrix with certain connection. Zhu et al. [14, 15] presented a hinge-free compliant mechanism which has one input and two outputs based on level set method. Jones [16] presented a wavelet-based topology optimization formulation by implicitly imbedding wavelet shrinkage method into optimization formulation based on SIMP method. Topology optimization methods for parallel compliant mechanisms may also be found in other literatures [17-19]. In those methods, compared with conventional parallel prototype mechanisms, the motion characteristics of designed structure are ambiguous. On the other hand, the mechanism has not included the hinge form after the topology optimization process. In this paper, we called this structure peristaltic structure.

In this paper, we proposed a new method for topology optimization of planar 3-DOF peristaltic structure parallel robot based on differential kinematic vector continuous mapping matrix with conventional prototype parallel mechanism. The remainder of the paper is organized as follows. In Section 2, the differential kinematic vector continuous mapping matrix with conventional prototype parallel mechanism is built. In Section 3, the optimization problems of design peristaltic structure are proposed based on SIMP method. The shape sensitivity analysis is applied to obtain the displacement field for the optimization combined with the vector mapping matrix. In Section 4, numerical examples are presented to demonstrate the effectiveness of the proposed method. Finally, conclusions and a discussion for further work are developed.

\section{Differential Kinematics with Vector Continuous Mapping Matrix}

In order to keep the differential kinematic characteristics with the prototype of parallel mechanism, the differential kinematic equations with vector continuous mapping of microelastic deformation mechanism should be set. Without loss of generality, we select the 3-RRR prototype of planar parallel mechanism as an example. As shown in Figure 1, the structure of 3-RRR prototype planar parallel mechanism is established.

Constraint equations are described as

$$
\begin{aligned}
& x_{B}=x_{A}+h \cdot \cos \varphi \\
& y_{B}=y_{A}+h \cdot \sin \varphi \\
& x_{C}=x_{A}+h \cdot \cos (\varphi+\pi) \\
& y_{C}=y_{A}+h \cdot \sin (\varphi+\pi) .
\end{aligned}
$$

Vector equation of the limb $\overline{P D A}$ may be described as

$$
\overrightarrow{O A}=\overrightarrow{O P}+\overrightarrow{P D}+\overrightarrow{D A}
$$

where $x_{p}=y_{p}=0$. Substituting (1) into (2), we obtain

$$
\begin{aligned}
& x_{A}=a_{1} \cdot \cos \theta_{1}+b_{1} \cdot \cos \left(\theta_{1}+\psi_{1}\right) \\
& y_{A}=a_{1} \cdot \sin \theta_{1}+b_{1} \cdot \sin \left(\theta_{1}+\psi_{1}\right) .
\end{aligned}
$$

Vector equation of the limb $\overline{B E Q}$ can be described as

$$
\overrightarrow{O B}=\overrightarrow{P Q}+\overrightarrow{Q E}+\overrightarrow{E B}
$$

where $x_{q}=c, y_{q}=0$. Substituting (1) into (4), we have

$$
\begin{aligned}
& x_{A}=c-a_{2} \cdot \cos \theta_{2}-b_{2} \cdot \cos \psi_{2}-h \cdot \cos \varphi \\
& y_{A}=a_{2} \cdot \sin \theta_{2}+b_{2} \cdot \sin \psi_{2}-h \cdot \sin \varphi \\
& x_{B}=c-a_{2} \cdot \cos \theta_{2}-b_{2} \cdot \cos \psi_{2} \\
& y_{B}=a_{2} \cdot \sin \theta_{2}+b_{2} \cdot \sin \psi_{2} .
\end{aligned}
$$


Vector equation of the limb $\overline{R F C}$ can be described as

$$
\overrightarrow{O C}=\overrightarrow{P C}+\overrightarrow{R F}+\overrightarrow{F C}
$$

where $x_{R}=c / 2, y_{R}=l$. Substituting (1) into (6) yields

$$
\begin{aligned}
& x_{A}=\frac{c}{2}-a_{3} \cdot \cos \theta_{3}-b_{3} \cdot \cos \psi_{3}-h \cdot \cos \left(\varphi+\frac{\pi}{3}\right) \\
& y_{A}=l-a_{3} \cdot \sin \theta_{3}-b_{3} \cdot \sin \psi_{3}-h \cdot \sin \left(\varphi+\frac{\pi}{3}\right) \\
& x_{C}=\frac{c}{2}-a_{3} \cdot \cos \theta_{3}-b_{3} \cdot \cos \psi_{3} \\
& y_{C}=l-a_{3} \cdot \sin \theta_{3}-b_{3} \cdot \sin \psi_{3} .
\end{aligned}
$$

We assumed that the displacements of each limb are equal to infinity and can be defined as $\theta_{i}^{\prime}=\theta_{i}+\Delta \theta_{i}, i=1,2,3$, and then (3), (5), and (7) can be rewritten as follows with infinite displacements $\mathbb{R}_{M}^{\prime}$ :

$$
\mathbb{R}_{M}^{\prime}=\left[\begin{array}{c}
x_{A}^{\prime} \\
y_{A}^{\prime} \\
x_{B}^{\prime} \\
y_{B}^{\prime} \\
x_{C}^{\prime} \\
y_{C}^{\prime}
\end{array}\right]
$$

$$
=\left[\begin{array}{c}
a_{1} \cdot \cos \left(\theta_{1}+\Delta \theta_{1}\right)+b_{1} \cdot \cos \left(\left(\theta_{1}+\psi_{1}\right)+\left(\Delta \theta_{1}+\Delta \psi_{1}\right)\right) \\
a_{1} \cdot \sin \left(\theta_{1}+\Delta \theta_{1}\right)+b_{1} \cdot \sin \left(\left(\theta_{1}+\psi_{1}\right)+\left(\Delta \theta_{1}+\Delta \psi_{1}\right)\right) \\
c-a_{2} \cdot \cos \left(\theta_{2}+\Delta \theta_{2}\right)-b_{2} \cdot \cos \left(\psi_{2}+\Delta \psi_{2}\right) \\
a_{2} \cdot \sin \left(\theta_{2}+\Delta \theta_{2}\right)+b_{2} \cdot \sin \left(\psi_{2}+\Delta \psi_{2}\right) \\
\frac{c}{2}-a_{3} \cdot \cos \left(\theta_{3}+\Delta \theta_{3}\right)-b_{3} \cdot \cos \left(\psi_{3}+\Delta \psi_{3}\right) \\
l-a_{3} \cdot \sin \left(\theta_{3}+\Delta \theta_{3}\right)-b_{3} \cdot \sin \left(\psi_{3}+\Delta \psi_{3}\right)
\end{array}\right] .
$$

Let $\Delta x=x_{A}^{\prime}-x_{A}$ and $\Delta y=y_{A}^{\prime}-y_{A}$, and the three parameters $\Delta x, \Delta y$, and $\Delta \varphi$ can be defined as output displacement of the moving platform.

The formulas of the active joints displacement and tasks displacement are included with (3), (5), (7), and (8), and the vector continuous mapping matrix between the joints displacement and task displacement can be expressed as follows:

$$
\begin{aligned}
& \Delta x \cdot \cos \left(\theta_{1}+\psi_{1}\right)+\Delta y \cdot \sin \left(\theta_{1}+\psi_{1}\right)-a_{1} \cdot \Delta \theta_{1} \\
& \quad \cdot \sin \psi_{1}=0 \\
& \Delta x \cdot \cos \psi_{2}+\Delta y \cdot \sin \psi_{2}+h \cdot \Delta \varphi \cdot \sin \left(\varphi+\psi_{2}\right)+a_{2} \\
& \quad \cdot \Delta \theta_{2} \cdot \sin \left(\theta_{2}-\psi_{2}\right)=0 \\
& \Delta x \cdot \cos \psi_{3}+\Delta y \cdot \sin \psi_{3}-h \cdot \Delta \varphi \cdot \sin \left(\varphi+\frac{\pi}{3}-\psi_{2}\right) \\
& \quad-a_{3} \cdot \Delta \theta_{3} \cdot \sin \left(\theta_{3}-\psi_{3}\right)=0 .
\end{aligned}
$$

Then the formulations can be rearranged and simplified as follows:

$$
\left[\begin{array}{l}
\Delta x \\
\Delta y \\
\Delta \varphi
\end{array}\right]=J_{D} \cdot\left[\begin{array}{l}
\Delta \theta_{1} \\
\Delta \theta_{2} \\
\Delta \theta_{3}
\end{array}\right],
$$

where

$$
\begin{aligned}
& J_{D}=\left[\begin{array}{ccc}
\frac{a_{1} \cdot s \psi_{1} \cdot\left(s(\alpha) \cdot s \psi_{2}+s(\gamma) \cdot s \psi_{3}\right)}{-M} & \frac{a_{2} \cdot s(\beta) \cdot s(\sigma) \cdot s(\alpha)}{-M} & \frac{a_{3} \cdot s(\beta) \cdot s(\gamma) \cdot s\left(\theta_{3}-\psi_{3}\right)}{-M} \\
\frac{a_{1} \cdot s \psi_{1} \cdot\left(s(\alpha) \cdot s \psi_{2} \cdot c \psi_{2}+s(\gamma) \cdot c \psi_{3}\right)}{M} & \frac{a_{2} \cdot c(\beta) \cdot s(\sigma) \cdot s(\alpha)}{M} & \frac{a_{3} \cdot c(\beta) \cdot s(\gamma) \cdot s\left(\theta_{3}-\psi_{3}\right)}{-M} \\
\frac{a_{1} \cdot s \psi_{1} \cdot s\left(\psi_{3}-\psi_{2}\right)}{h \cdot M} & \frac{a_{2} \cdot s\left(\theta_{2}-\psi_{2}\right) \cdot s\left(\psi_{3}-\theta_{1}-\psi_{1}\right)}{h \cdot M} & \frac{a_{3} \cdot s\left(\theta_{3}-\psi_{3}\right) \cdot s\left(\psi_{2}-\theta_{1}-\psi_{1}\right)}{h \cdot M}
\end{array}\right], \\
& \alpha=\varphi+\frac{\pi}{3}-\psi_{3} \\
& \beta=\theta_{1}+\psi_{1} \\
& \gamma=\varphi+\psi_{2} \\
& \sigma=\theta_{2}-\psi_{2} \\
& M=s\left(\varphi+\frac{\pi}{3}-\psi_{3}\right) \cdot s\left(\theta_{1}+\psi_{1}-\psi_{2}\right)+s\left(\varphi+\psi_{2}\right) \cdot s\left(\theta_{1}+\psi_{1}-\psi_{3}\right) \\
& s(*)=\sin (*), \\
& c(*)=\cos (*) .
\end{aligned}
$$




\section{Topology Optimization of 3-DOF Peristaltic Structure}

3.1. SIMP Model of Topology Optimization with Vector Continuous Mapping Matrix. The kinematic characteristics of 3DOF peristaltic structure have three input parameters and three output parameters, respectively. By using the vector continuous mapping matrix, the model of SIMP can be built as

$$
\begin{aligned}
& \min \quad C=\sum_{i=1}^{3} \sum_{j=1}^{3}\left(\widetilde{U}_{j}^{T} K U_{i}\right)=\sum_{\substack{1 \leq e \leq N \\
1 \leq i \leq 3 \\
1 \leq j \leq 3}} \widetilde{U}_{e j}^{T} \rho_{e}^{p} K_{E} U_{e i} \\
& \text { s.t. } \quad\left\{K \widetilde{U}_{j}=F_{j}, K U_{i}=F_{i}, F_{j}=J_{D} F_{i}\right\} ; \\
& \quad i=1,2,3 ; j=1,2,3 \\
& \forall: \longrightarrow \int_{\Omega} \rho_{e} d \Omega \leq V, \\
& 0 \leq \rho_{\min } \leq \rho_{e} \leq 1 ; e=1,2, \ldots, N .
\end{aligned}
$$

In (12) $U_{j}$ is the companion displacement vector, $K$ is the whole postoptimality stiffness of creep structure, $U_{i}$ is the displacement with actual load, $V$ is postoptimality volume of creep structure, $F_{i}$ is $i$ th actual load, $U_{e i}$ is the unit displacement vector under the $i$ th actual load, $F_{i}$ is $j$ th virtual load, $\widetilde{U}_{e i}$ is the unit displacement vector under the $j$ th virtual load, $K_{E}$ is unit stiffness, $\rho_{e}$ is unit density, and $p$ is penalty factor and satisfies $p \geq \max \left\{2 /\left(1-V^{0}\right), 4 /\left(1+v^{0}\right)\right\}$.

3.2. Sensitivity Analysis. Sensitivity analysis in topology optimization includes the sensitivity of objective function and constraint function. Differentiating the two types of functions with respect to design variables, we can derive the direction of iterative update during topology optimization process.

3.2.1. Sensitivity Analysis of Objective Function. Differentiating formulation (12) with respect to density $\rho_{e}$ yields

$$
\begin{aligned}
& \frac{\partial C}{\partial \rho_{e}}=\frac{\partial\left(\sum_{i=1}^{3} \sum_{j=1}^{3}\left(\widetilde{U}_{j}^{T} K U_{i}\right)\right)}{\partial \rho_{e}} \\
& =\frac{\partial\left(\sum_{j=1}^{3} \widetilde{U}_{j}^{T}\right)}{\partial \rho_{e}} \cdot K \cdot \sum_{i=1}^{3} U_{i}+\sum_{j=1}^{3} \widetilde{U}_{j}^{T} \cdot \frac{\partial K}{\partial \rho_{e}} \cdot \sum_{i=1}^{3} U_{i} \\
& +\sum_{j=1}^{3} \widetilde{U}_{j}^{T} \cdot K \cdot \frac{\partial\left(\sum_{i=1}^{3} U_{i}\right)}{\partial \rho_{e}} \\
& =\left(\frac{\partial\left(\sum_{j=1}^{3} \widetilde{U}_{j}^{T}\right)}{\partial \rho_{e}} \cdot K+\sum_{j=1}^{3} \widetilde{U}_{j}^{T} \cdot \frac{\partial K}{\partial \rho_{e}}\right) \cdot \sum_{i=1}^{3} U_{i} \\
& \rho_{e}^{(k+1)}= \begin{cases}\min \left\{(m+1) \rho_{e}^{(k)}, 1\right\} & \text { s.t. } \min \left\{(1+m) \cdot \rho_{e}^{(k)}, 1\right\} \leq\left(D_{e}^{(k)}\right)^{\zeta} \cdot \rho_{e}^{(k)} \\
\left(D_{e}^{(k)}\right)^{\zeta} \cdot \rho_{e}^{(k)} & \text { s.t. } \max \left\{(1-m) \cdot \rho_{e}^{(k)}, \rho_{\min }\right\}<\left(D_{e}^{(k)}\right)^{\zeta} \cdot \rho_{e}^{(k)}<\min \left\{(1+m) \cdot \rho_{e}^{(k)}, 1\right\} \\
\max \left\{(1-m) \rho_{e}^{(k)}, \rho_{\min }\right\} & \text { s.t. }\left(D_{e}^{(k)}\right)^{\zeta} \cdot \rho_{e}^{(k)} \leq \max \left\{(1-m) \cdot \rho_{e}^{(k)}, \rho_{\min }\right\},\end{cases}
\end{aligned}
$$

From a macro perspective, without loss of generality, we assumed that the input and output are unconcerned with design variables, and then differentiating formulation (12) with respect to design variables, we obtain

$$
\begin{gathered}
\frac{\partial K}{\partial \rho_{e}} \cdot \sum_{j=1}^{3} \widetilde{U}_{j}^{T}+K \cdot \frac{\partial\left(\sum_{j=1}^{3} \widetilde{U}_{j}^{T}\right)}{\partial \rho_{e}}=\frac{\partial\left(\sum_{j=1}^{3} F_{j}\right)}{\partial \rho_{e}}=0 \\
\frac{\partial K}{\partial \rho_{e}} \cdot \sum_{i=1}^{3} U_{i}+K \cdot \frac{\partial\left(\sum_{i=1}^{3} U_{i}\right)}{\partial \rho_{e}}=\frac{\partial\left(\sum_{i=1}^{3} F_{i}\right)}{\partial \rho_{e}}=0 .
\end{gathered}
$$

Substituting $K=\sum_{e=1}^{N} \rho_{e}^{p} \cdot K_{E}$ into (15), the computational formula for objective function sensitivity analysis is given by

$$
\frac{\partial C}{\partial \rho_{e}}=\sum_{e=1}^{N} \sum_{i=1}^{3} \sum_{j=1}^{3} p \widetilde{U}_{j}^{T} \rho_{e}^{p-1} K_{E} U_{i} .
$$

3.2.2. Sensitivity Analysis of Constraint Function. Differentiating the volume function with respect to the unit density $\rho_{e}$, sensitivity of constraint function can be derived as

$$
\frac{\partial V}{\partial \rho_{e}}=\frac{\partial\left(\int_{\Omega} \rho_{e} d V\right)}{\partial \rho_{e}}=1 .
$$




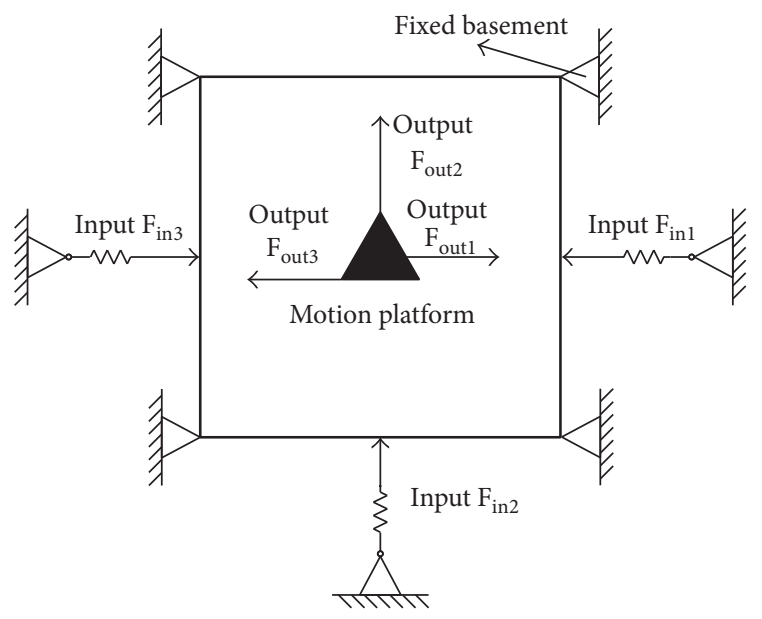

(a)

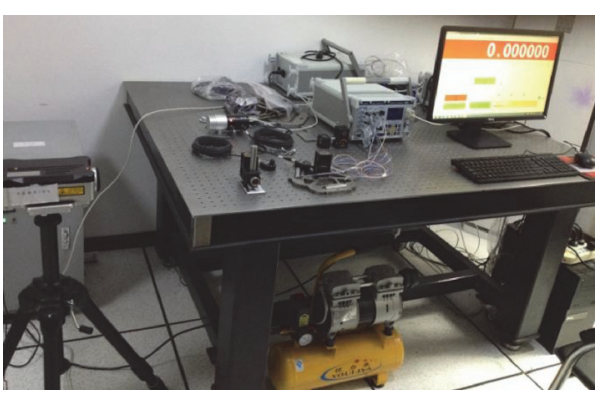

(b)

FIGURE 2: Design conditions of topology optimization for 3-DOF robot of peristaltic structure (a) and the experimental mechanism (b).

where $\rho_{e}^{(k)}$ is the iteration value of $k$ step, $\rho_{e}^{(k+1)}$ is the iteration value of $k+1$ step, and $m$ is the moving limit constant.

$m$ denotes the appropriate constraint to design variables for stabilizing the iteration process. Based on literature [10], the range of parameter $m$ may be selected within $0.1 \sim 0.3$. $\zeta$ is damping factor and in the range of $0.4 \sim 0.5$, and $D_{e}^{(k)}$ is expressed as follows:

$$
\begin{aligned}
D_{e}^{(k)} & =\frac{p \rho_{e}^{(p-1)} \sum_{j=1}^{3} \widetilde{U}_{j}^{T} \cdot K_{E} \cdot \sum_{i=1}^{3} U_{i} \cdot \Delta E}{\Lambda^{(k)} V_{e}} \\
& =\frac{\left(\max \left(0,-\partial C / \partial \rho_{e}\right)\right)}{\Lambda^{(k)} V_{e}}
\end{aligned}
$$

where $\Lambda^{(k)}$ is the Lagrange multiplier with volume constraint of $k$ step iteration and the update of Lagrange multiplier is adapted double convex linear programming algorithm. And $V_{e}$ is the unit volume of $k$ step iteration.

3.4. Heaviside Filter. We adapt Heaviside function to modify the linear filter for peristaltic structure in 3-DOF robot. As the range of minimum filter radius, if $\widetilde{\rho}_{e}>0$, then $\bar{\rho}_{e}=1$; otherwise, $\tilde{\rho}_{e}=0$; then $\bar{\rho}_{e}=0$. The resulting expression of linear filter can be given by

$$
\tilde{\rho}_{i}=\sum_{j} \omega_{i j} \rho_{j}, \quad \sum_{j} \omega_{i j}=1,
$$

where $\omega_{i j}$ is filter weight factor of the $j$ th unit to the $i$ th unit.

To get the better discrete 0/1 distribution optimization results and decent middle density unit, second weight calculation equation and Heaviside function are adopted as follows:

$$
\omega_{i j}= \begin{cases}\frac{r_{\text {min }}-d(i, j)}{\sum_{k \in N_{i}}\left(r_{\min }-d(i, k)\right)} & j \in N_{i} \\ 0 & j \notin N_{i} \\ N_{i}=\left\{j: r_{\min }-d(i, k) \geq 0\right\}, & \end{cases}
$$

where $d(i, j)$ is the displacement between $i$ th unit and $j$ th unit and $r_{\min }$ is the minimum filter radius.

We adapt again the Heaviside function to approximate the original linear density filter. The Heaviside filter function may be expressed as

$$
\bar{\rho}_{e}=1+\exp \left(-\beta \cdot \widetilde{\rho}_{e}\right)+\widetilde{\rho}_{e} \cdot \exp (-\beta),
$$

where $\beta$ is the control parameter for flat degree of Heaviside function. If $\beta=0$, then Heaviside filter can be changed to linear filter; if $\beta=\infty$, Heaviside filter can be changed to maximum density filter. $\bar{\rho}_{e}$ is unit physical density, and $\tilde{\rho}_{e}$ is linear result of unit density.

Heaviside function filter can be freely switched between linear density filter and maximum density filter in the method of parameters selection.

\section{Design Conditions of Topology Optimization for 3-DOF Peristaltic Structure}

Based on linear elastic relationship between force and displacement, the $3 \mathrm{DOF}$ of peristaltic structure have been changed to force output, as shown in Figure 2. $F_{\text {in } 1}, F_{\text {in } 2}$, and $F_{\text {in3 }}$ are the force inputs in the fixed basement, and $F_{\text {out } 1}, F_{\text {out } 2}$, and $F_{\text {out3 }}$ are the force outputs in the motion platform center.

\section{Simulations and Experimental Results Analysis of 3-DOF Peristaltic Structure Robot}

Assuming that the topology optimization yield of peristaltic structure is a square profile with $14 \times 14$ unit size and the number of discrete grids is $140 \times 140$, the elastic modulus of solid material is $2 \times 10^{11} \mathrm{~Pa}$, the elastic modulus of holes is $10^{-3} \mathrm{~Pa}$, Poisson's ratio is 0.35 , optimum volume ratio is 0.278 , minimum filter radius is 1.4 , input force $F_{\text {in } 1}$ is $1200 \mathrm{~N}$, 


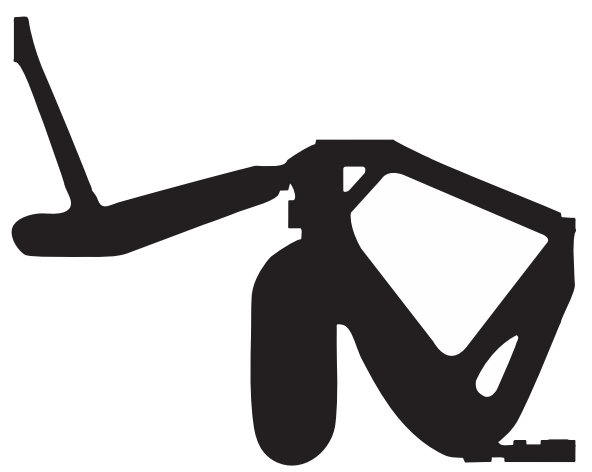

FIGURE 3: The topological structure of 3-DOF peristaltic structure robot optimization result.

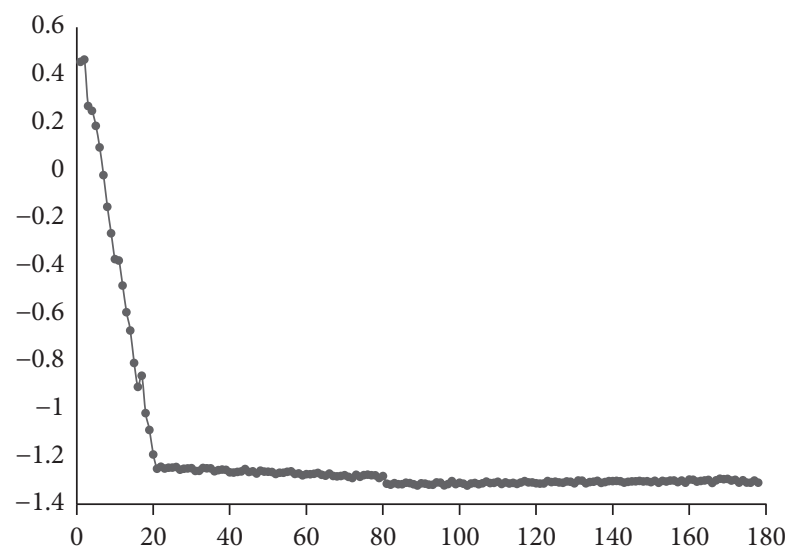

FIGURE 4: Objective function $C$ and convergence history of 3-DOF peristaltic structure switched curve.

input force $F_{\text {in2 }}$ is $1200 \mathrm{~N}$, and input force $F_{\text {in3 }}$ is $1200 \mathrm{~N}$. Based on the relationship between input forces and output forces denoted as $J_{D}, F_{j}=J_{D} \cdot F_{i}$, the output forces can be solved as $F_{\text {out } 1}=478 \mathrm{~N}, F_{\text {out } 2}=1570 \mathrm{~N}$, and $F_{\text {out } 3}=1240 \mathrm{~N}$, respectively. The optimization was run for 180 iterations, and the topology optimized result of 3-DOF peristaltic structure robot is shown in Figure 3, and the iterative process of the objective function $C$ optimization is shown in Figure 4.

We adapt the general curve fitting for the contour of 3-DOF peristaltic structure robot and the median filtering method to smooth the boundary of peristaltic structure optimized result. Import the contour data into SolidWorks ${ }^{\circledR}$ 3D software; the modeling structure is shown in Figure 5.

And then, CAE software by ANSYS $^{\circledR}$ is used for static simulations of 3-DOF peristaltic structure robot. Import the 3D model of the optimized result into ANSYS software, dividing finite element mesh and setting boundary conditions, and carry on static analysis. The simulations method of 3-DOF peristaltic structure robot is shown in Figure 6. According to the previous conditions set, the fixed basement is in the diagonal of the simulation domain. The input forces $F_{1}, F_{2}$, and $F_{3}$ in acting points of 3-DOF peristaltic structure robot are transferred to the output region (the moving platform) in its elastic deformation method, including the displacement of direction $x / y$ and rotational direction $z$.

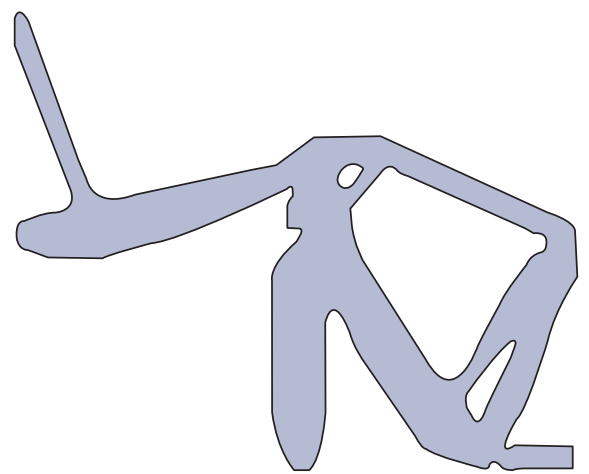

FIGURE 5: Modeling of 3-DOF peristaltic structure robot with median filtering method.

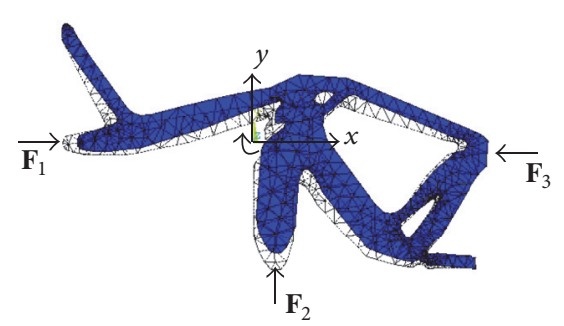

FIGURE 6: 3-DOF peristaltic structure robot static simulations analysis.

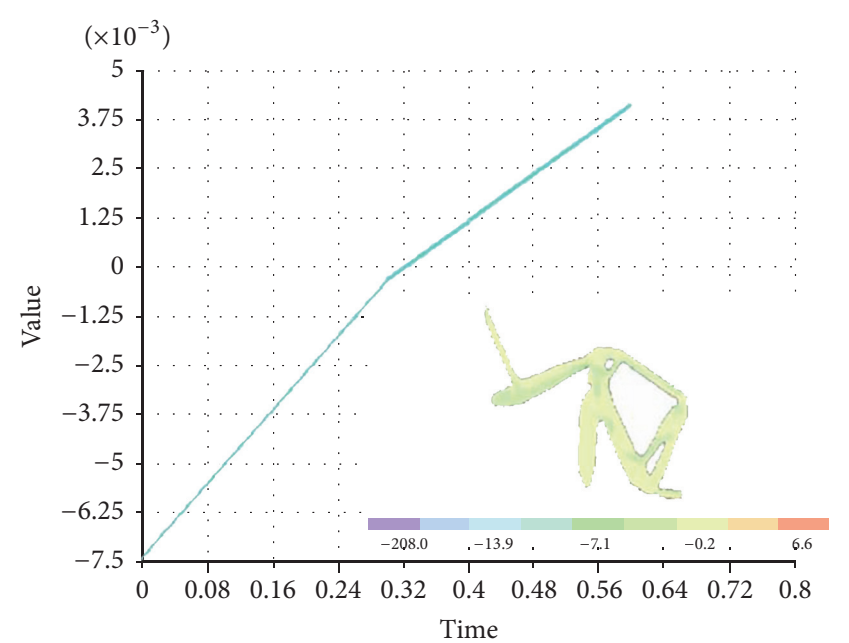

FIGURE 7: Differential displacement of 3-DOF peristaltic structure robot with direction $x$.

The differential displacement of directions $x$ and $y$ in the center of moving platform is shown in Figures 7 and 8 with the maximum values $-2.15 \mathrm{~nm}$ and $1.80 \mathrm{~nm}$, respectively. The differential rotational displacement of direction $z$ is shown in Figure 9 with the maximum value $-4.15 \mu \mathrm{rad}$.

According to Section 2, 3-DOF peristaltic structure differential kinematic analysis, we set initial condition in certain steady state in Table 1 . 
TABLE 1: 3-DOF peristaltic structure differential kinematic parameters.

\begin{tabular}{|c|c|c|c|c|c|c|c|c|c|c|}
\hline$\theta_{1}$ & $\theta_{2}$ & $\theta_{3}$ & $\psi_{1}$ & $\psi_{2}$ & $\psi_{3}$ & $\varphi$ & $a_{1}$ & $a_{2}$ & $a_{3}$ & $h$ \\
\hline$\frac{2}{3} \pi$ & $\frac{4}{9} \pi$ & $\frac{1}{6} \pi$ & $\frac{1}{12} \pi$ & $\frac{1}{9} \pi$ & $\frac{1}{3} \pi$ & $\frac{5}{36} \pi$ & 6 & 6 & 6 & 10 \\
\hline
\end{tabular}

TABLE 2: Differential displacements of the 3-DOF peristaltic structure robot in theory and simulation.

\begin{tabular}{lccc}
\hline & Differential displacement of $x$ & Differential displacement of $y$ & Differential displacement of $z$ \\
\hline Value of theory & $-2.15 \mathrm{~nm}$ & $1.80 \mathrm{~nm}$ & $-4.15 \mu \mathrm{rad}$ \\
Value of simulation & $-6.15 \mathrm{~nm}$ & $2.93 \mathrm{~nm}$ & $-3.55 \mu \mathrm{rad}$ \\
\hline
\end{tabular}

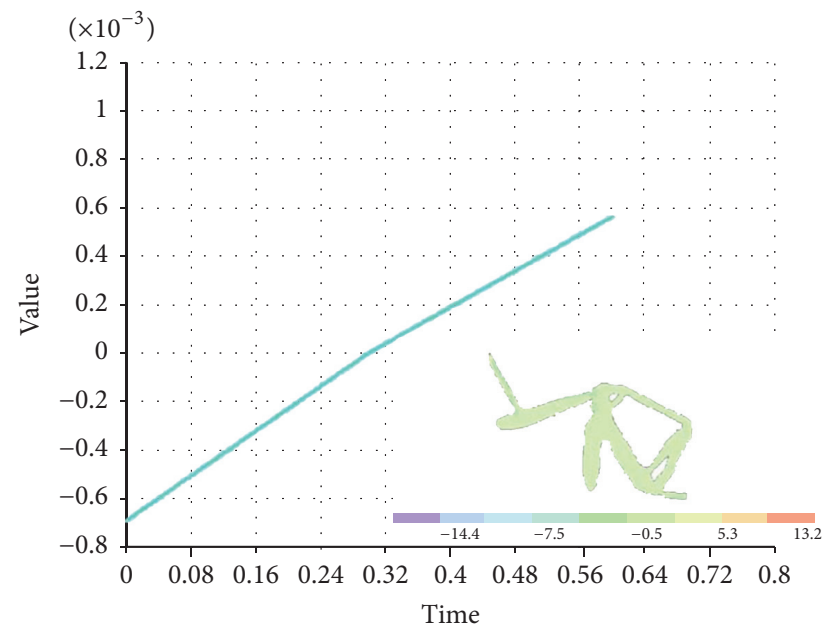

FIGURE 8: Differential displacement of 3-DOF peristaltic structure robot with direction $y$.

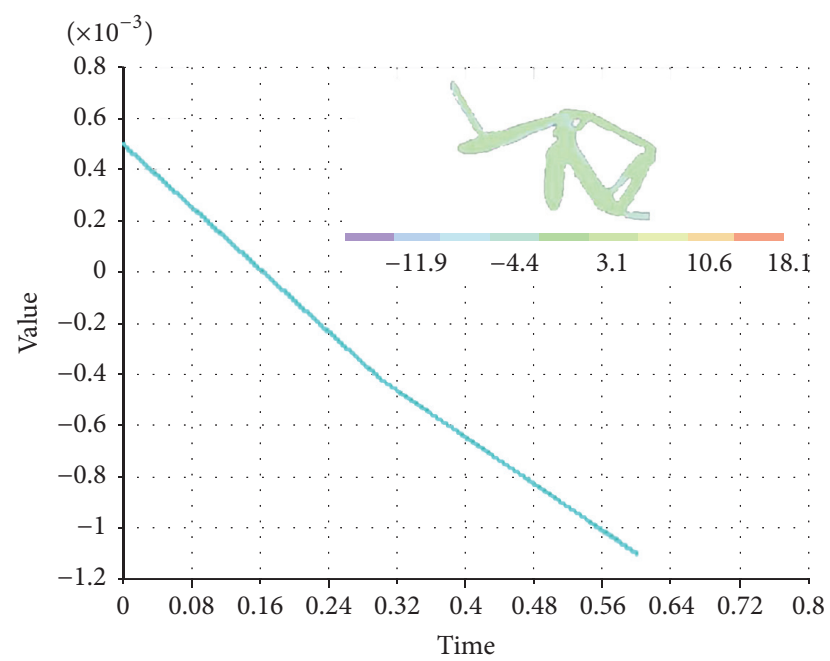

FIGURE 9: Differential displacement of 3-DOF peristaltic structure robot with direction $z$.

Substituting these parameters shown in Table 1 into (10), we obtain

$$
\left[\begin{array}{ccc}
-2.0109 & -2.3827 & 2.5432 \\
2.1459 & -0.6673 & 0.2989 \\
0.9943 & -0.1023 & -0.6925
\end{array}\right]\left[\begin{array}{c}
\Delta \theta_{1} \\
\Delta \theta_{2} \\
\Delta \theta_{3}
\end{array}\right]=\left[\begin{array}{c}
\Delta x \\
\Delta y \\
\Delta \varphi
\end{array}\right]
$$

Substituting input parameters $\left(\begin{array}{lll}\Delta \theta_{1} & \Delta \theta_{2} & \Delta \theta_{3}\end{array}\right)=$ (2.5 2.5 2.5) into (23), the displacement of moving platform may be given by

$$
\left[\begin{array}{l}
\Delta x \\
\Delta y \\
\Delta \varphi
\end{array}\right]=\left(\begin{array}{lll}
-6.15 & 2.93 & -3.55
\end{array}\right)^{\prime} .
$$

Comparing the simulation results in Figures 7 9 with theoretical calculation results (24) in the same computational scale, the proposed 3-DOF peristaltic structure robot no longer suffers the high stress concentration and is more suitable for real world applications, as shown in Table 2.

From Table 2, we can see that the results of orders of magnitude and symbols are the same in all directions/rotations, and the differential displacement of 3-DOF peristaltic structure has the same characteristic with the parallel prototype mechanism. The peristaltic structure robot realizes 3-DOF planar kinematics, which is displacement $x / y$ and rotational displacement $z$ in micron level. So simulations verify the correctness of vector continuous mapping topology optimization design method.

However, we note that the quantitative errors between the values of theory and simulation are so big, and analysis results of error source are shown in two factors:

(1) The theoretical result and simulation result adopt the structure of 3-RRR prototype planar parallel mechanism and the 3-DOF peristaltic structure robot, respectively. The former transfers force and kinematics through rigid hinges, and the latter transfers force and kinematics through its own elastic deformation. Due to elastic potential energy in the latter own inside, the output force $F_{j}$ displacement decreases.

(2) We assumed the elastic deformation and displacement outputs approximate linearization and the stress/ strain transfer in peristaltic structure is isotropic.

\section{Conclusions}

In this paper, we presented a new formulation for synthesis of peristaltic structure with vector continuous mapping matrix based on topology optimization method. In the proposed method, the vector continuous mapping matrix, derived with 3-RRR parallel prototype mechanism, is taken into account to make the peristaltic structure to inherit the same 
kinematic characteristic with parallel prototype mechanism. Consequently, we proposed a new topology optimization method combining SIMP with vector continuous mapping matrix to synthesise 3-DOF peristaltic structure robot. Adopt the Heaviside filter to modify optimization function dependence of mesh grid division. We use curve fitting method to smooth the contour for optimization result and modeling 3D peristaltic structure. Import the model into ANSYS and implement static simulations analysis. Numerical simulations with vector continuous mapping matrix are presented for illustrating the validity of the presented method. Some conclusions are obtained:

(1) 3-DOF peristaltic structure robot moving platform realizes $x$-displacement $-6.15 \mathrm{~nm}, y$-displacement $2.93 \mathrm{~nm}$, and $z$-rotational displacement $-3.55 \mu \mathrm{rad}$ and shows planar 3-DOF parallel mechanism differential kinematics.

(2) Compared with similar general parallel mechanism kinematics, in approximately equal load conditions, the results of orders of magnitude and symbols are the same in all directions/rotations. The 3-DOF peristaltic structure robot based on vector continuous mapping matrix design method delivers the same kinematic characteristic with similar general parallel mechanism, which qualitatively verifies validity of the method.

\section{Competing Interests}

The authors declare that they have no competing interests.

\section{Acknowledgments}

This work was supported by the National Natural Science Foundation of China under Grant no. 51165009, China Postdoctoral Science Foundation under Grant no. 2013M541874, Science and Technology Project of Guangdong Province (China) under Grant no. 2013B011302002, and Science and Technology Program of Guangzhou (China) under Grant no. $201508010025 / 2015$ Y2000010. The authors would like to thank Vanta Intelligent Equipment Technology Co., Ltd., in Guangzhou for supporting them with the experimental setup.

\section{References}

[1] L. L. Howell, Compliant Mechanisms, John Wiley \& Sons, New York, NY, USA, 2013.

[2] W. Bejgerowski, J. W. Gerdes, S. K. Gupta, and H. A. Bruck, "Design and fabrication of miniature compliant hinges for multi-material compliant mechanisms," International Journal of Advanced Manufacturing Technology, vol. 57, no. 5-8, pp. 437452, 2011.

[3] Y. Yun and Y. Li, "Design and analysis of a novel 6-DOF redundant actuated parallel robot with compliant hinges for high precision positioning," Nonlinear Dynamics, vol. 61, no. 4 , pp. 829-845, 2010.
[4] W. Dong, Z. Du, L. Sun, and B. Zheng, "A compliant ultra-precision 6-DOF parallel positioner based on the coarse/fine dual architecture," in Proceedings of the 1st IEEE International Conference on Nano/Micro Engineered and Molecular Systems, pp. 488-492, Zhuhai, China, January 2006.

[5] K.-B. Choi, J. J. Lee, G. H. Kim, and H. J. Lim, "A compliant parallel mechanism with flexure-based joint chains for two translations," International Journal of Precision Engineering and Manufacturing, vol. 13, no. 9, pp. 1625-1632, 2012.

[6] Z. Gao and D. Zhang, "Simulation driven performance characterization of a spatial compliant parallel mechanism," International Journal of Mechanics and Materials in Design, vol. 10, no. 3, pp. 227-246, 2014.

[7] Z. Dachang and F. Yanping, "Structure design of a 3-DOF UPC type rotational fully spatial compliant parallel manipulators," International Journal of Advancements in Computing Technology, vol. 5, no. 8, pp. 70-81, 2013.

[8] D. Zhang and Z. Gao, "Performance analysis and optimization of a five-degrees-of-freedom compliant hybrid parallel micromanipulator," Robotics and Computer-Integrated Manufacturing, vol. 34, pp. 20-29, 2015.

[9] G. Hao and H. Li, "Design of 3-legged XYZ compliant parallel manipulators with minimised parasitic rotations," Robotica, vol. 33, no. 4, pp. 787-806, 2015.

[10] M. P. Bendsøe and N. Kikuchi, "Generating optimal topologies in structural design using a homogenization method," Computer Methods in Applied Mechanics and Engineering, vol. 71, no. 2, pp. 197-224, 1988.

[11] M. P. Bendsøe and O. Sigmund, "Material interpolation schemes in topology optimization," Archive of Applied Mechanics, vol. 69, no. 9-10, pp. 635-654, 1999.

[12] M. I. Frecker, G. K. Ananthasuresh, S. Nishiwaki, N. Kikuchi, and S. Kota, "Topological synthesis of compliant mechanisms using multi-criteria optimization," Journal of Mechanical Design, vol. 119, no. 2, pp. 238-245, 1997.

[13] G. Allaire, F. Jouve, and A.-M. Toader, "Structural optimization using sensitivity analysis and a level-set method," Journal of Computational Physics, vol. 194, no. 1, pp. 363-393, 2004.

[14] B. Zhu, X. Zhang, and N. Wang, "Topology optimization of hinge-free compliant mechanisms with multiple outputs using level set method," Structural and Multidisciplinary Optimization, vol. 47, no. 5, pp. 659-672, 2013.

[15] B. Zhu, X. Zhang, and S. Fatikow, "A multi-objective method of hinge-free compliant mechanism optimization," Structural and Multidisciplinary Optimization, vol. 49, no. 3, pp. 431-440, 2014.

[16] J. Jones, Contact Mechanics, chapter 6, Cambridge University Press, Cambridge, UK, 2000.

[17] G. Hao and H. Li, "Conceptual designs of multi-degree of freedom compliant parallel manipulators composed of wire-beam based compliant mechanisms," Proceedings of the Institution of Mechanical Engineers, Part C: Journal of Mechanical Engineering Science, vol. 229, no. 3, pp. 538-555, 2015.

[18] G. Z. Lum, T. J. Teo, G. Yang, S. H. Yeo, and M. Sitti, "Integrating mechanism synthesis and topological optimization technique for stiffness-oriented design of a three degrees-of-freedom flexure-based parallel mechanism," Precision Engineering, vol. 39, pp. 125-133, 2015.

[19] J. Beroz, A. J. Hart, and S. Awtar, "Extensible-link kinematic model for characterizing and optimizing compliant mechanism motion," Journal of Mechanical Design, vol. 136, no. 3, Article ID 031008, 2014. 


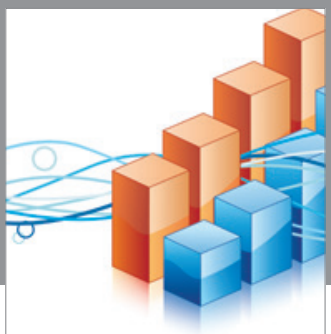

Advances in

Operations Research

vatem alat4

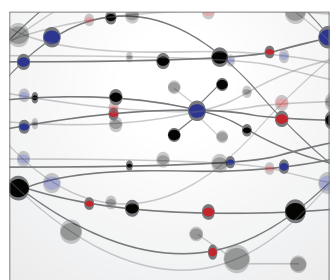

\section{The Scientific} World Journal
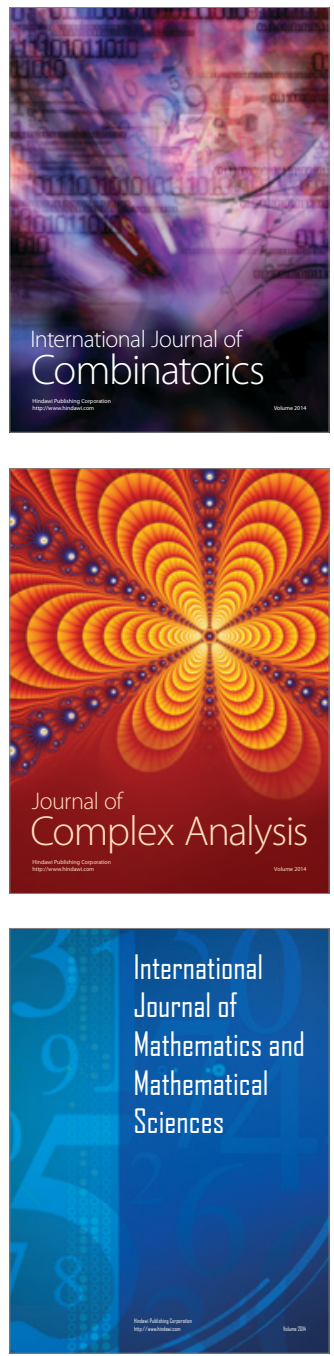
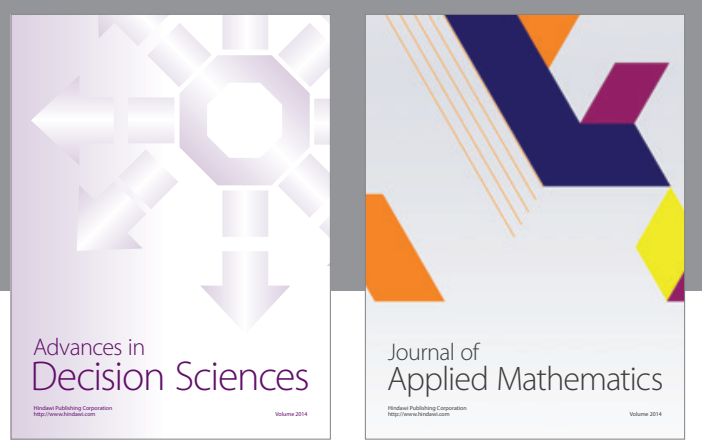

Algebra

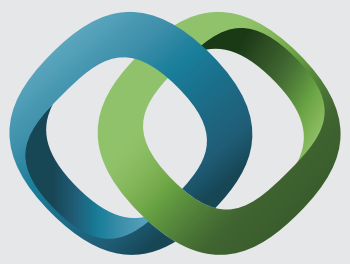

\section{Hindawi}

Submit your manuscripts at

http://www.hindawi.com
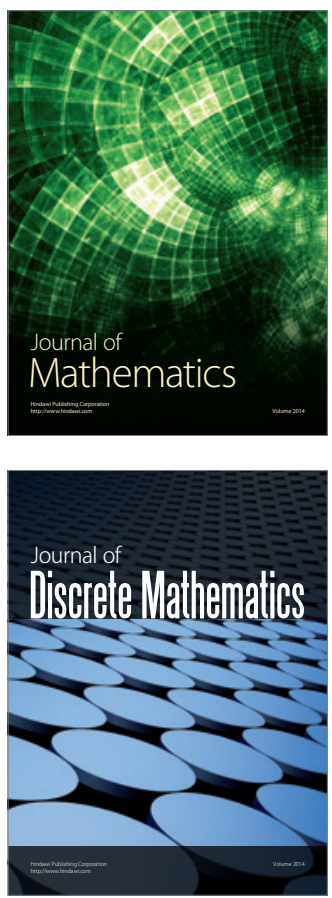

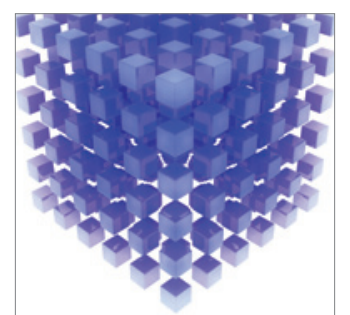

Mathematical Problems in Engineering
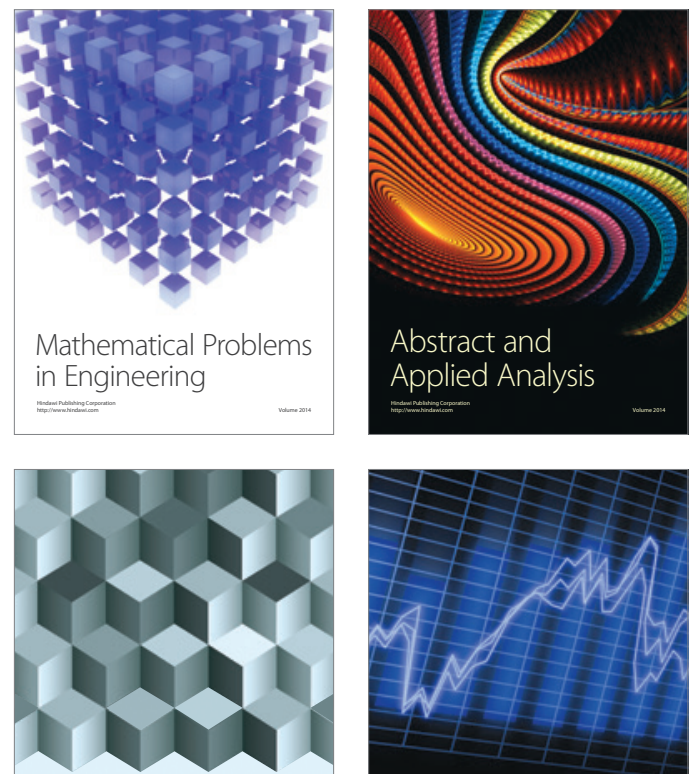

Journal of

Function Spaces

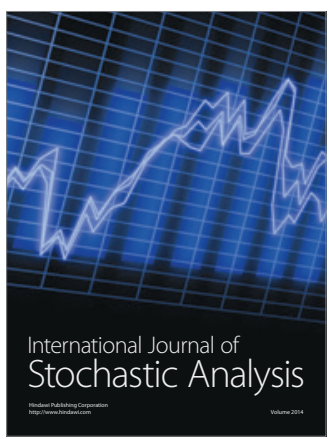

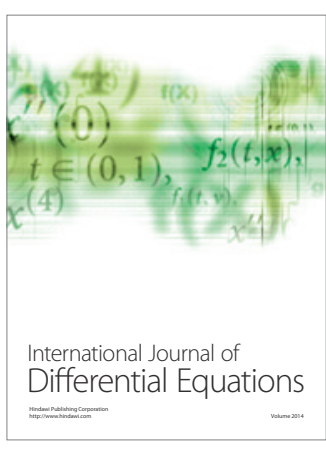
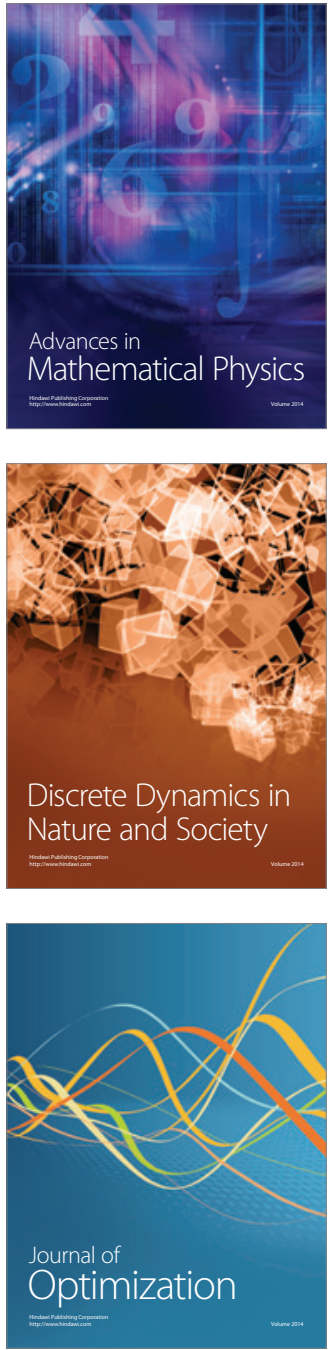